\title{
Phase correlation functions: FFT vs. FHT
}

\author{
Giuseppe Schirripa Spagnolo ${ }^{1}$, Lorenzo Cozzella ${ }^{1}$, Fabio Leccese ${ }^{2}$ \\ ${ }^{1}$ Università Roma Tre - Dipartimento di Matematica e Fisica, Via della Vasca Navale, 84, 00146 Roma, Italy \\ 2 Università Roma Tre - Dipartimento di Scienze, Via della Vasca Navale, 84, 00146 Roma, Italy
}

\section{ABSTRACT}

The ability to process an image is a crucial skill in many measurement activities. In image processing or pattern recognition, Fast Fourier Transform (FFT) is widely used. In particular, the Phase Only Correlation (POC) method demonstrates high robustness and subpixel accuracy in pattern matching. However, there is a disadvantage in the required memory machine because of the calculation of 2D-FFT. In applications in which the use of memory is a critical element, Fast Hartley Transform (FHT) seems to be a good substitute. In this context, the use of Hartley's transform can be of interest for apps implemented on portable systems e.g. smartphones. In this article, we present a comparison of the implementations of the phase correlation function using FFT and FHT. Particular attention is given to the analytical steps necessary to implement the POC by means of the Hartley transform.

\section{Section: RESEARCH PAPER}

Keywords: FFT; Phase Correlation; Fast Hartley Transform; Digital Image Elaboration

Citation: G. Schirripa Spagnolo, L. Cozzella, F. Leccese, Phase correlation functions: FFT vs. FHT, Acta IMEKO, vol. 8, no. 1, article 12, March 2019, identifier: IMEKO-ACTA-08 (2019)-01-12

Editor: Alistair Forbes, National Physical Laboratory, UK

Received May 05, 2018; In final form March 20, 2019; Published March 2019

Copyright: @ 2019 IMEKO. This is an open-access article distributed under the terms of the Creative Commons Attribution 3.0 License, which permits unrestricted use, distribution, and reproduction in any medium, provided the original author and source are credited.

Funding: This work was supported by MIUR.

Corresponding author: F. Leccese, e-mail: fabio.leccese@uniroma3.it

\section{INTRODUCTION}

The ability to accurately process images and video signals is an important skill in many research fields. One of the more widespread techniques used is the Fourier transform [1], [2]. In fact, Fourier's theorem is not only one of the most impressive results of analysis and one of the most important mathematical tools available to physicists and engineers, but it may be considered an indispensable instrument in digital image processing. In this field, the Fourier's transform and correlationbased analysis are crucial elements of the pattern-matching process [3], [4]. In many image-processing applications, it is necessary to determine the correspondence between two or more images. The different images can either be images of the same object taken from different sensors or images of the same object taken at different times. This matching analysis is typically performed using either cross-correlation in the spatial domain or phase correlation in the frequency domain. In general, spatial cross-correlation [5], [6] yields several broad peaks and a main peak whose maximum is not always clearly defined; therefore, it is difficult to evaluate and locate it. On the other hand, correlation in the frequency domain is preferable in the case that the images are corrupted by noise.
Figure 1 shows the correlation function between two images, one of which is corrupted by noise.

In this figure, it is possible to note the difference between the cross-correlation in the spatial domain and the phase correlation performed in the frequency domain.

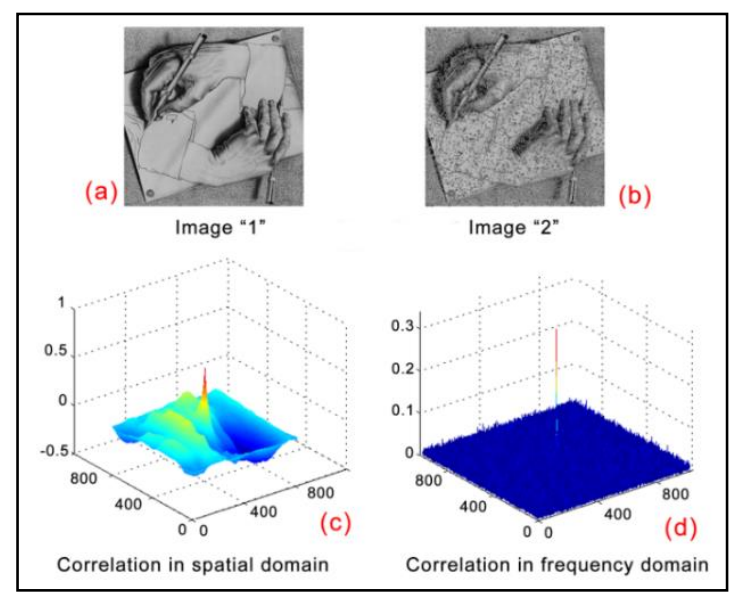

Figure 1. Example of correlation between two images (a) Image ' 1 '; (b) Image ' 2 '; (c) correlation in the spatial domain; (d) correlation in frequency domain. 
The phase correlation in the frequency domain is efficiently performed by Phase-Only Correlation (POC) [7], [8]. This correlation method demonstrates high robustness and accuracy in pattern matching and in image registration. However, there is a disadvantage in terms of the required machine memory because of the calculation of 2D-FFT. The Fast Hartley Transform (FHT) can be a valid alternative to the Fast Fourier Transform (FFT) [9]. The Hartley transform resembles a Fourier transform, but it is free from the need to process complex numbers. The Hartley transform also has better properties and faster algorithms than the Fourier one; therefore, it can represent a valid alternative, particularly useful when a greater efficiency in memory requirements is needed.

In this paper, we analyse the equivalence of the POC computation by means of FFT and FHT. The Hartley transform, in some cases, is a valid alternative to the Fourier transform, in particular in all those applications implemented on embedded systems or smartphones.

The remaining of this paper is organised as follows. The definition of the POC function and its basic properties are described in section 2. The properties of the Harley transform and the definition of POC in Hartley space are given in section 3. In section 4 , we present a set of experiments for evaluating the performance of the proposed methods. Finally, conclusions are drawn in section 5 .

\section{PHASE-ONLY CORRELATION (POC)}

POC is a digital image processing technique that is normally used to estimate the 'similarity' between two images. It is extensively used in many practical applications. In particular, POC is employed in image registration [10]-[12], fingerprint matching [13], [14], iris recognition [15], palmar recognition [16], PIV analysis [17], digital image stabilisation [18], and security applications [19]-[24].

Phase correlation is based on the well-known Fourier shift property [25]. If we consider two $N_{x} \times N_{y}$ digital images $I_{1}(x, y)$ and $I_{2}(x, y)$. We assume that the index ranges are $x=-M_{x}, \ldots, M_{x}$, and $y=-M_{y}, \ldots, M_{y}$ for mathematical simplicity. Hence, $N_{x}=2 M_{x}+1$ and $N_{y}=2 M_{y}+1$. Let $F_{1}(u, v)$ and $F_{2}(u, v)$ denote the $2 \mathrm{D}$ Discrete Fourier Transforms of the two images $I_{1}(x, y)$ and $I_{2}(x, y)$.

$F_{1}(u, v)$ and $F_{2}(u, v)$ are given by:

$$
\begin{aligned}
& F_{1}(u, v)=\frac{1}{N_{x} N_{y}} \sum_{x=-M_{x}}^{M_{x}} \sum_{y=-M_{y}}^{M_{y}} I_{1}(x, y) \exp \left[-j 2 \pi\left(\frac{u x}{N_{x}}+\frac{v y}{N_{y}}\right)\right] \\
& F_{2}(u, v)=\frac{1}{N_{x} N_{y}} \sum_{x=-M_{x}}^{M_{x}} \sum_{y=-M_{y}}^{M_{y}} I_{2}(x, y) \exp \left[-j 2 \pi\left(\frac{u x}{N_{x}}+\frac{v y}{N_{y}}\right)\right]
\end{aligned}
$$

where $u=-M_{x}, \ldots, M_{x}, v=-M_{y}, \ldots, M_{y}$

The cross-phase spectrum (or normalised cross spectrum) is defined as [26]

$$
\frac{F_{1}(u, v) \cdot F_{2}^{*}(u, v)}{\left|F_{1}(u, v) \cdot F_{2}^{*}(u, v)\right|}=\exp [-j(u x+v y)]
$$

with $F_{2}^{*}(u, v)$ being the conjugate function of $F_{2}(u, v)$. The POC function is the $2 \mathrm{D}$ inverse discrete Fourier transform of equation (3). In other words, the POC surface is defined as $r(x, y)=F^{-1}\left[\frac{F_{1}(u, v) \cdot F_{2}^{*}(u, v)}{\left|F_{1}(u, v) \cdot F_{2}^{*}(u, v)\right|}\right]$

If both images are similar, the POC function $r(x, y)$ gives a distinct sharp peak. Conversely, if the two images are not similar, the peak significantly drops. The height of the peak gives a similarity measurement of the matching, and the position of the peak in the $x$ e $y$ axis shows the translation displacement of two images. The most remarkable property of POC compared to the ordinary correlation is that it is not influenced by brightness change, and it is highly robust against noise.

POC, in some cases, is defined as [27], [28]

$r(x, y)=F^{-1}\left[\frac{F_{1}(u, v) \cdot F_{2}^{*}(u, v)}{\left|F_{1}(u, v) \cdot F_{2}^{*}(u, v)\right|^{\alpha}}\right]$

In equation (5), the coefficient $\alpha$ controls the correlation peak width. Optimum values range from $\alpha=0$ (for images characterised by high spatial frequency content and a high noise level) to $\alpha=0.5$ (for low-noise images with a less fine structure). For values greater than 0.5 , the high frequency noise is magnified [29].

Figure 2 shows two examples of POC outputs.

In some applications, instead of FFT, it is possible to use FHT. Using FHT, equation (4) and equation (5) can be computed efficiently and without loss of information [30].

\section{THE HARTLEY TRANSFORM}

The Hartley transform, introduced in 1942 [31], became widely known after the publication of Bracewell's study in 1983 [32].

It is an integral transform closely related to the Fourier transform; keeps all the useful properties thereof; and can be used to obtain the power spectrum and perform convolution directly through the output real-valued data without firstly calculating the real and imaginary parts. The use of the Hartley transform also reduces the processing time and needs less memory. Therefore, it is particularly well suited for application on mobile devices and embedded systems.

The Hartley transform is one type of integral transformation that is closely related to the Fourier transform. It has the same integral core of the transform and the inverse transform, and it also has most of the characteristics of the Fourier transform.

The Hartley transform can be obtained from the Fourier integral by replacing the exponential function

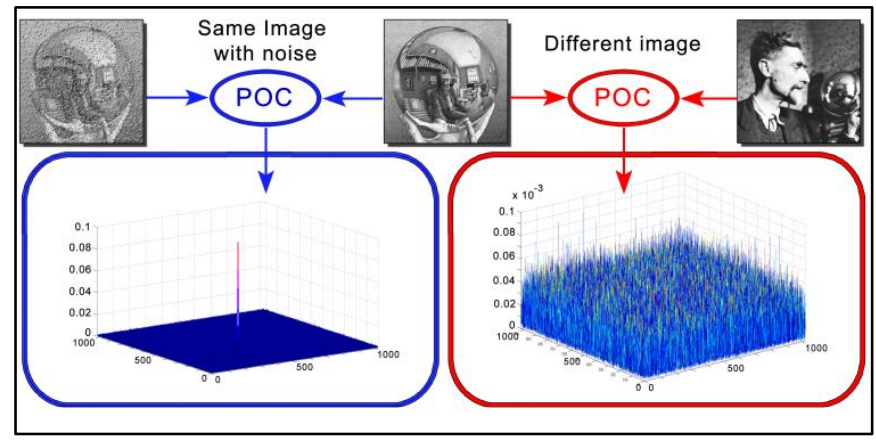

Figure 2. Examples of the POC function. 
$\exp [-j 2 \pi(u x+v y)]=\cos [2 \pi(u x+v y)]-j \sin [2 \pi(u x+v y)]$

with $\operatorname{cas}[2 \pi(u x+v y)]=\cos (2 \pi u x)+\sin (2 \pi u x)$

The Hartley transform $S_{H}(u, v)$ of a real signal $s(x, y)$ and its inverse transform are defined as

$S_{H}(u, v)=\int_{-\infty}^{+\infty} \int_{-\infty}^{+\infty} s(x, y) \operatorname{cas}[2 \pi(u x+v y)] d x d y$

$s(x, y)=\int_{-\infty}^{+\infty} \int_{-\infty}^{+\infty} S_{H}(x, y) \operatorname{cas}[2 \pi(u x+v y)] d u d v$.

The Fourier and Hartley transforms are very similar and share many properties. Consequently, many applications using the Fourier transform can be performed by the Hartley one [33].

To derive the relationship between the Fourier and Hartley transforms, their symmetry must be considered. Let us split the Hartley transform $S_{H}(u, v)$ into its even and odd parts $E_{H}(u, v)$ and $O_{H}(u, v)$, so that

$S_{H}(u, v)=E_{H}(u, v)+O_{H}(u, v)$.

The even part of the function is what we get by reversing the function (changing $u$ to $-u$ and $v$ to $-v$ ), adding the reversed function to the original and dividing by two, that is

$E_{H}(u, v)=\frac{S_{H}(u, v)+S_{H}(-u, v)}{2}$.

The odd part is formed by subtracting the reversed function and dividing by two:

$O_{H}(u, v)=\frac{S_{H}(u, v)-S_{H}(-u, v)}{2}$.

Any function may be split uniquely into even and odd parts and from the even and odd parts, if given, the original function may be uniquely reconstituted.

Obviously, the Fourier transform can also be divided into even and odd parts: $E_{F}(u, v)$ and $O_{F}(u, v)$. Furthermore,

$E_{F}(u, v)=\operatorname{Re}\left[S_{F}(u, v)\right]$

$O_{F}(u, v)=\operatorname{Im}\left[S_{F}(u, v)\right]$.

By considering the definitions of the Hartley and Fourier transforms, we have $E_{H}(u, v)=E_{F}(u, v)$ and $O_{H}(u, v)=-O_{F}(u, v)$. Therefore,

$$
\begin{aligned}
S_{H}(u, v) & =\operatorname{Re}\left[S_{F}(u, v)\right]-\operatorname{Im}\left[S_{F}(u, v)\right], \\
S_{F}(u, v) & =E_{F}(u, v)+j O_{F}(u, v)=E_{H}(u, v)-j O_{H}(u, v) \\
& =\frac{1}{2}\left\{\left[S_{H}(u, v)+S_{H}(-u,-v)\right]\right. \\
& \left.-j\left[S_{H}(u, v)-S_{H}(-u,-v)\right]\right\} .
\end{aligned}
$$

The generalisation of splitting single-variable functions into even and odd parts is the decomposition into symmetric and antisymmetric parts

$$
g(x, y)=g_{\text {symm }}(x, y)+g_{\text {antisymm }}(x, y)
$$

with

$$
g_{\text {symm }}(x, y)=\frac{1}{2}[g(x, y)+g(-x,-y)],
$$

$$
g_{\text {antisymm }}(x, y)=\frac{1}{2}[g(x, y)-g(-x,-y)] .
$$

The real part of a 2D Fourier transform is symmetrical, and the imaginary part is antisymmetric. Therefore,

$E_{H}(u, v)=\frac{G_{H}(u, v)+G_{H}(-u,-v)}{2}$,

$O_{H}(u, v)=\frac{G_{H}(u, v)-G_{H}(-u,-v)}{2}$

with $G_{F}(u, v)=E_{H}(u, v)-j O_{H}(u, v)$, which is the $2 \mathrm{D}$ extension of equation (14)

The cross-correlation $r(x, y)$ of the two functions $p(x, y)$ and $q(x, y)$ is

$r(x, y)=\int_{-\infty}^{+\infty} \int_{-\infty}^{+\infty} p(x, y) q\left(x+x^{\prime}, y+y^{\prime}\right) d x d y$,

where $x^{\prime}$ and $y^{\prime}$ are variable shift components along the $x$ direction and the $y$-direction respectively.

Evaluating the cross-correlation by means of the integral in equation (20) is complicated. This task can be significantly simplified in the Fourier space, where $R_{F}(u, v)=P_{F}^{*}(u, v) \cdot Q_{F}(u, v)$; with $R_{F}(u, v), \quad P_{F}(u, v) \quad$ and $Q_{F}(u, v)$ being the Fourier transforms of $r(x, y), p(x, y)$, and $q(x, y)$, respectively. Therefore,

$r(x, y)=\mathrm{FFT}^{-1}\left\{R_{F}\right\}=\mathrm{FFT}^{-1}\left\{P_{F}^{*}(u, v) \cdot Q_{F}(u, v)\right\}$.

In general, the presence of noise makes the 'exact' localisation of the cross-correlation peak difficult. In other words, using the POC function,

$r(x, y)=\mathrm{FFT}^{-1}\left\{\frac{R_{F}(u, v)}{\left|R_{F}(u, v)\right|}\right\}$

and we obtain a good compromise between peak sharpness and noise tolerance.

Using the Hartley transform, $R_{F}$ can be written as [34]

$$
\begin{aligned}
R_{F}(u, v)= & \frac{R_{H}(u, v)+R_{H}(-u,-v)}{2}-i \frac{R_{H}(u, v)-R_{H}(-u,-v)}{2} \\
= & {\left[\frac{P_{H}(u, v)+P_{H}(-u,-v)}{2}+i \frac{P_{H}(u, v)-P_{H}(-u,-v)}{2}\right] } \\
& \cdot\left[\frac{Q_{H}(u, v)+Q_{H}(-u,-v)}{2}-i \frac{Q_{H}(u, v)-Q_{H}(-u,-v)}{2}\right] \\
= & \frac{P_{H}(u, v) \cdot Q_{H}(u, v)+P_{H}(-u,-v) \cdot Q_{H}(-u,-v)}{2} \\
& +j \frac{P_{H}(u, v) \cdot Q_{H}(-u,-v)-P_{H}(-u,-v) \cdot Q_{H}(u, v)}{2}
\end{aligned}
$$

where $R_{H}(u, v)$ is the Hartley transform of $r(x, y)$. From equation (23), we obtain

$$
\begin{aligned}
& R_{H}(u, v)+R_{H}(-u,-v) \\
& \quad=P_{H}(u, v) \cdot Q_{H}(u, v)-P_{H}(u, v) \cdot Q_{H}(-u,-v) \\
& R_{H}(u, v)-R_{H}(-u,-v) \\
& \quad=P_{H}(-u,-v) \cdot Q_{H}(u, v)-P_{H}(u, v) \cdot Q_{H}(-u,-v)
\end{aligned}
$$

and eventually 


$$
\begin{aligned}
R_{H}(u, v)= & \frac{P_{H}(u, v) \cdot Q_{H}(u, v)-P_{H}(u, v) \cdot Q_{H}(-u,-v)}{2} \\
& +\frac{P_{H}(-u,-v) \cdot Q_{H}(u, v)+P_{H}(-u,-v) \cdot Q_{H}(-u,-v)}{2}
\end{aligned}
$$

Therefore, the cross-correlation can be computed by means of the Hartley transform. Indicating with FHT, we have

$$
\begin{aligned}
r(x, y)= & \mathrm{FHT}^{-1}\left\{R_{H}\right\} \\
= & \mathrm{FHT}^{-1}\left\{\frac{P_{H}(u, v) \cdot Q_{H}(u, v)-P_{H}(u, v) \cdot Q_{H}(-u,-v)}{2}\right. \\
& \left.+\frac{P_{H}(-u,-v) \cdot Q_{H}(u, v)+P_{H}(-u,-v) \cdot Q_{H}(-u,-v)}{2}\right\}
\end{aligned}
$$

Introducing the POC function, we have

$$
r(x, y)=\mathrm{FHT}^{-1}\left\{\frac{R_{H}(u, v)}{\left|R_{H}(u, v)\right|}\right\} .
$$

\section{RESULTS AND DISCUSSION}

A number of FHT algorithms have been developed [9], [35][39]. To test the proposed technique, an FHT was implemented by using the algorithms reported in [40]. Furthermore, the application of the Hartley POC function, in comparison with Fourier POC, was tested.

Figure 3 shows the POC obtained by two fingerprint patterns. In this example, the two images are each $512 \times 512$ pixels. The POC was computed in the Fourier space by means of equation (22) and in the Hartley space by means of equation (27). The FFT POC and the FHT POC are very similar, virtually identical.

In banknotes and other documents, metal fibres are present, as security features, inside the paper pulp. These fibres can only be observed under ultraviolet light. After image enhancement and some segmentation processes, it is possible to obtain a binary controlling grid that indicates the position of the majority security fibres. The distribution of these metallic fibres is random and not reproducible; each banknote can be told apart from the

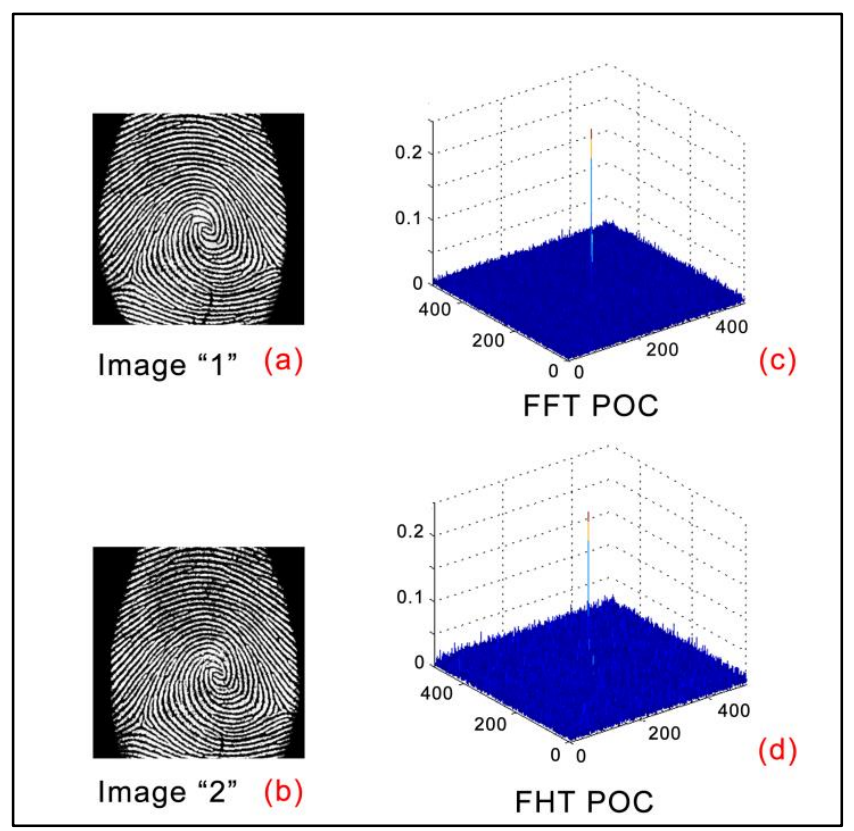

Figure 3. Examples of the POC function obtained from two fingerprint images.

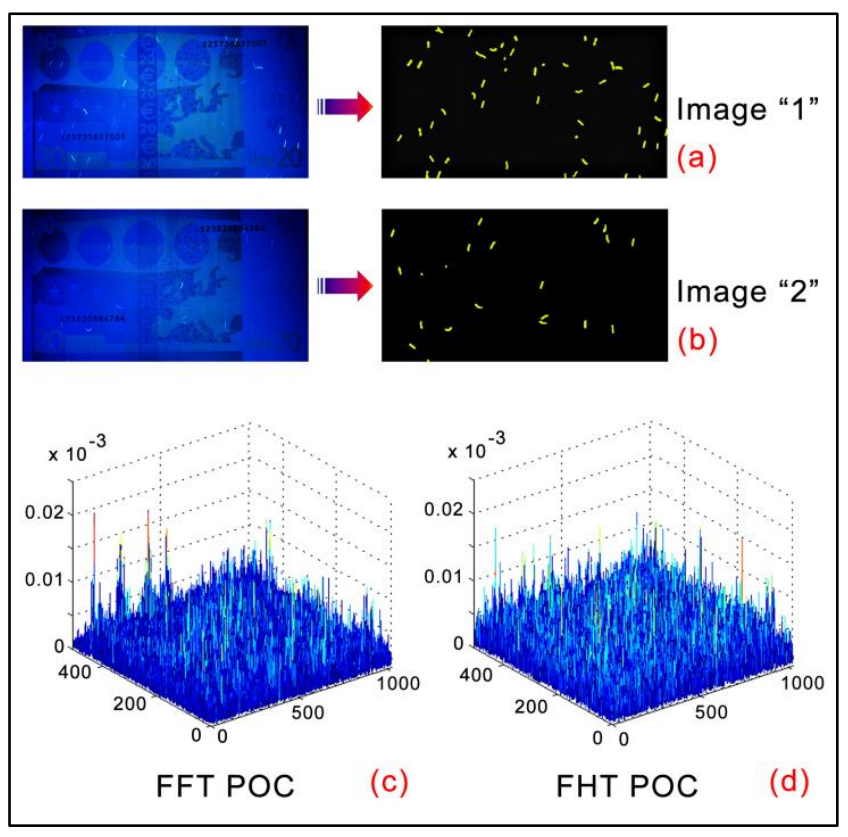

Figure 4. Examples of the POC function obtained from two different banknotes. In this example, the two images are each $512 \times 1024$ pixels.

others using this random distribution. Obviously, the captured image has residual geometrical distortion and noise. Therefore, a verification approach based on digital phase correlation calculation can be a good solution [20], [21].

Figure 4 shows the POC used in the banknote identification. In this example, the fluorescent fibres (embedded in the paper) of two different 20-Euro banknotes are compared.

The POC functions obtained by means of FFT and FHT is practically identical. The examples illustrated do not prove that the FHT is superior to the FFT, but they do demonstrate that the FHT is fully compatible with the FFT.

Figure 5 shows an example of a drug package watermark realised with florescent points printed with ultraviolent lightsensitive ink. This watermark has a structure similar to the one used in speckle measurement [41]. In this example, the robustness of the POC approach against geometrical distortions and watermark partial disruption (also in the case of the Hartley transform POC) is highlighted. In particular, the correct watermark identification is highly relevant even in case c), where more than half of the watermark was unavailable.

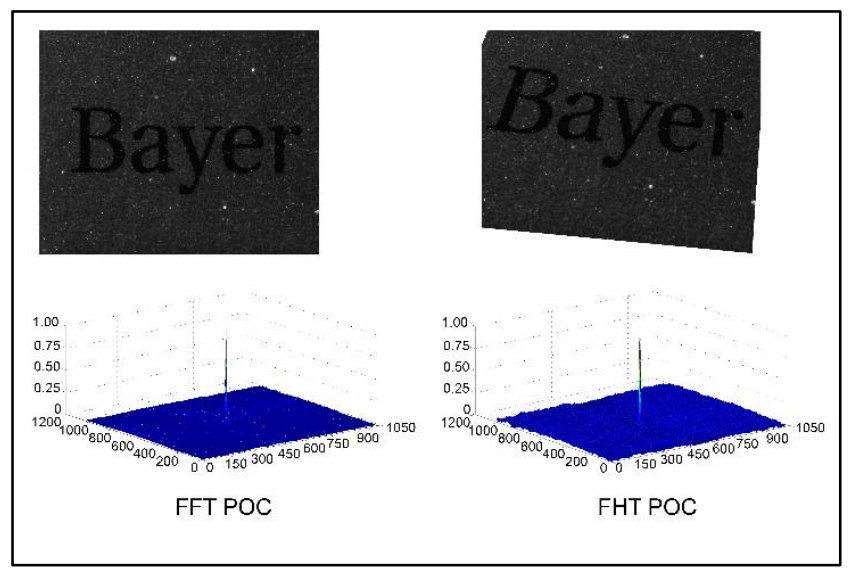

Figure 5. Examples of the POC functions obtained from two different acquisitions of drug package watermarks. In this example, it is possible to note the robustness of the POC approach against geometrical distortions. 
In general, to perform FFT and FHT, the same number of operations are required. In other words, FHT requires a comparable number of steps to execute and is of comparable complexity to the FFT.

In any case, the FHT algorithm requires only real arithmetic computations compared to complex arithmetic operations in any standard FFT. Therefore, the speed of performing an FHT should be about twice as fast as the FFT. However, it is hard to compare the run times of these two programs for the transform process itself because the programs used in this effort are implemented differently.

On the other hand, the use of only real-value functions implies fewer computational resources and less computer memory; usually, the memory requirement by typical FFT algorithm is twice as high as for FHT.

\section{CONCLUSION}

In this paper, we have shown the correspondence between the POC function obtained by means of FFT and FHT.

The Hartley transform is similar to the Fourier transform, but it is free from the need to process complex numbers. Furthermore, the FHT performs the transformation itself, and the convolution and the cross-correlation are undertaken with fewer additions and multiplications than FFT. Using FHT to compute cross-correlation, there is no loss of information, and the results are identical to those obtained by FFT. Unfortunately, the numbers of all other operations are the same. Differences in time consuming exist between FHT and complex-valued FFT, and in any case, even compared with newly real-valued FFT algorithms, the possibility to use an identical programming solution for forward and inverse transformation is still an important point for using FHT instead of FFT in image correlation. In addition, the fact that the Hartley transform does not use complex numbers leads to the need for less memory to store numbers than the Fourier one. The Hartley transform can therefore be considered a valid alternative to FFT, particularly useful when a greater efficiency in memory requirements is needed, as, for instance, in embedded systems and smartphone apps.

\section{REFERENCES}

[1] S. Bochner, K. Chandrasekharan, Fourier Transforms, Annals of Mathematics Studies (AM-19), Princeton University Press, 41 William Street, Princeton, New Jersey, 08540, USA, 1950, ISBN: 9780691095783

[2] R. N. Bracewell, The Fourier Transform \& Its Applications, 3 $3^{\text {rd }}$ edn, McGraw-Hill Higher Education, Boston, 2000, ISBN 9780073039381.

[3] R. O. Duda, P. E. Hart, Pattern Classification and Scene Analysis, Wiley, New York, 1973, ISBN: 978-0471223610.

[4] R. C. Gonzalez, R. E. Woods, Digital Image Processing, 3 $3^{\text {rd }}$ edn, Addison-Wesley, Reading, Massachusetts, 1992, ISBN: 978-0131687288.

[5] N. Wiener, Extrapolation, Interpolation, and Smoothing of Stationary Time Series, M.I.T. Press, Cambridge, Massachusetts, 1949, ISBN: 9780262230025.

[6] J. Y. Stein, Digital Signal Processing: A Computer Science Perspective, Wiley, New York, 2000, ISBN: 978-0471295464.

[7] J. L. Horner, P. D. Gianino Phase-only matched filtering, Applied Optics 23, 6 (1984), pp. 812-816, DOI: https://doi.org/10.1364/AO.23.000812.

[8] B. S. Reddy, B. N. Chatterji, An FFT-based technique for translation, rotation, and scale-invariant image registration, IEEE Trans. on Image Processing 5,8 (1996) pp. 1266-1271, doi: https://doi.org/10.1109/83.506761.

[9] F. Piccinin, The fast Hartley transform as an alternative to the fast Fourier transform: technical memorandum SRL-000-TM, Department of Defence, Defence Science and Technology Organization, Salisbury Surveillance Research Laboratory, South Australia, 1988,

http://www.dtic.mil/dtic/tr/fulltext/u2/a212493.pdf [Access date: 18.04 .2018$]$.

[10] Q. S. Chen, M. Defrise, F. Deconinck, Symmetric phase-only matched filtering of Fourier-Mellin transform for image registration and recognition, IEEE Trans. on Pattern Analysis and Machine Intelligence 16, 12 (1994) pp. 1156-1168, DOI: https://doi.org/10.1109/34.387491.

[11] K. Takita, T. Aoki, Y. Sasaki, T. Higuchi, K. Kobayashi, Highaccuracy subpixel image registration based on phase-only correlation, IEICE Trans. Fundamentals E86-A(8) 2003, pp. 1925-1934, https://pdfs.semanticscholar.org/8a6d/91acae1b18b66846a1 d211887d551dca5e58.pdf [Access date: 18.04.2018].

[12] Y. Keller, A. Averbuch, A projection-based extension to phase correlation image alignment, Signal Processing 87, 1 (2007) pp. 124-133, DOI: https://doi.org/10.1016/i.sigpro.2006.04.013.

[13] K. Ito, H. Nakajima, K. Kobayashi, T. Aoki, T. Higuchi, A fingerprint matching algorithm using phase-only correlation, IEICE Trans. Fundamentals E87-A(3) 2004, pp. 682-691, https://pdfs.semanticscholar.org/87e6/585eb46d92a16256b0 b9ba5e3eb19ce603b5.pdf [Access date: 18.04.2018].

[14] F. Francis-Lothai, D. B. L. Bong, A fingerprint matching algorithm using bit-plane extraction method with phase-only correlation, Int. J. Biometrics 9, 1 (2017) pp. 44-66, DOI: https://doi.org/10.1504/IJBM.2017.084135.

[15] K. Miyazawa, K. Ito, T. Aoki, K. Kobayashi, H. Nakajima, An effective approach for iris recognition using phase-based image matching, IEEE Trans. on Pattern Analysis and Machine Intelligence 30, 10 (2008), pp. 1741-1756,

DOI: https://doi.org/10.1109/TPAMI.2007.70833.

[16] K. Ito, T. Aoki, H. Nakajima, K. Kobayashi, T. Higuchi, A palmprint recognition algorithm using phase-based image matching', Proc. of the $13^{\text {th }}$ IEEE Int'l Conf. Image Processing, Atlanta, USA, 8 - 11 October 2006, pp. 2669-2672,

DOI: https://doi.org/10.1109/ICIP.2006.313059.

[17] M. Thomas, S. Misra, C. Kambhamettu, J. T. Kirby, A robust motion estimation algorithm for PIV, Meas. Sci. Technol. 16, 3 (2005) pp. 865-877, DOI: https://doi.org/10.1088/0957-0233/16/3/031.

[18] S. Erturk, 'Digital image stabilization with sub-image phase correlation-based global motion estimation', IEEE Trans. on Consumer Electronics 49, 4 (2003), pp. 1320-1325, DOI: https://doi.org/10.1109/TCE.2003.1261235.

[19] G. Schirripa Spagnolo, L. Cozzella, C. Simonetti, Banknote security using a biometric-like technique: a hylemetric approach, Meas. Sci. Technol. 21 (2010), pp. 55501-55509, DOI: https://doi.org/10.1088/0957-0233/21/5/055501.

[20] G. Schirripa Spagnolo, L. Cozzella, C. Simonetti, Currency verification by a $2 \mathrm{D}$ infrared barcode, Meas. Sci. Technol. 21 (2010) 21, pp. 107002-107006, DOI: https://doi.org/10.1088/0957-0233/21/10/107002.

[21] L. Cozzella, C. Simonetti, G. Schirripa Spagnolo, Drug packaging security by means of white-light speckle, Optics and Lasers in Engineering 50 (2012), pp. 1359-1371,

DOI: https://doi.org/10.1016/j.optlaseng.2012.05.016.

[22] G. Schirripa Spagnolo, L. Cozzella, D. Papalillo, Smartphone sensors for stone lithography authentication, Sensors 1 (2014), pp. 8217-8234, DOI: https://doi.org/10.3390/s140508217.

[23] L. Cozzella, G. Schirripa Spagnolo, F. Leccese, Biometric-like approach for verifying artworks authenticity, Applied Physics Research 5, 6 (2013), pp. 118-130, 
DOI: https://doi.org/10.5539/apr.v5n6p118.

[24] G. Schirripa Spagnolo, L. Cozzella, M. Caciotta, R. Colasanti, G. Ferrari, Painting authentication by means of a biometric-like approach, ACTA IMEKO 4 (2015) 3, pp. 65-71, DOI: https://doi.org/10.21014/acta imeko.v4i3.260.

[25] R. N. Bracewell, The Fourier Transform and Its Applications, 3rd edn, McGraw-Hill Higher Education, Boston, 2000, ISBN 9780073039381.

[26] M. Sjödahl, L. R. Benckert, Electronic speckle photography: analysis of an algorithm giving the displacement with subpixel accuracy, Appl. Opt. 32, 13 (1993), pp. 2278-2284, DOI: https://doi.org/10.1364/AO.32.002278.

[27] M. Sjödahl, Digital speckle photography, in: Trends in Optical Non-Destructive Testing and Inspection, P. K. Rastogi and D. Inaudi (editors), Elsevier Publishing, Amsterdam, 2000, pp. 179-195, ISBN 9780080430201.

[28] D. Amodio, G. B. Broggiato, F. Campana, G. M. Newaz, Digital speckle correlation for strain measurement by image analysis, Experimental Mechanics 43, 4 (2003), pp. 396-402, DOI: https://doi.org/10.1007/BF02411344.

[29] D. J. Chen, F. P. Chiang, Y. S. Tan, H. S. Don, Digital speckledisplacement measurement using a complex spectrum method, Appl. Opt. 32, 11 (1993), pp. 1839-1849,

DOI: https://doi.org/10.1364/AO.32.001839.

[30] L. Cozzella, G. Schirripa Spagnolo, Phase-only correlation function by means of Hartley transform, JSM Math. Stat. 1, 1 (2014), p. 1004-1011,

https://pdfs.semanticscholar.org/945f/5a9bfa6fb1f617290f7 6472addda57a20fca.pdf [Access date: 18.04.2018].

[31] R. V. L. Hartley, A more symmetrical Fourier analysis applied to transmission problems, Proc. of the IRE, 30, 3, New York, USA, 7 October 1942, pp. 144-150,

DOI: https://doi.org/10.1109/JRPROC.1942.234333.

[32] R. N. Bracewell, Discrete Hartley transform, JOSA 73, 12 (1943), pp. 1832-1835, DOI: https://doi.org/10.1364/JOSA.73.001832.
[33] R. N. Bracewell, The Hartley Transform, Oxford University Press, New York, 1986, ISBN: 9780195039696.

[34] H. Sun, The Hartley transform applied to particle image velocimetry, Meas. Sci. Technol. 13(12) (2002), pp. 1996-2000, DOI: https://doi.org/10.1088/0957-0233/13/12/326.

[35] D. J. Lee, M. Ramirez, S. Sunanda Mitra, Fast 2-D Hartley transform in 3-D object representation and recognition, Proc. of SPIE vol. 1608, 1992, Intelligent Robots and Computer Vision X: Neural, Biological, and 3-D Methods, 1 March, 1992 Boston, MA, USA,

DOI: https://doi.org/10.1117/12.135097.

[36] H. J. Meckelburg, D. Lipka, Fast Hartley transform algorithm, Electronics Letters 21, 8 (1985), pp. 341-343, DOI: https://doi.org/10.1049/el:19850241.

[37] M. Popovic, D. Sevic, A new look at the comparison of the fast Hartley and Fourier transforms, IEEE Trans. on Signal Processing 42, 8 (1994), pp. 2178-2182, DOI: https://doi.org/10.1109/78.301854.

[38] H. Bantikyan, Implementation of parallel fast Hartley transform (FHT) using Cuda, Journal of Computer Sciences and Applications 2, 1 (2014), pp. 6-8,

DOI: https://doi.org/10.12691/jcsa-2-1-2.

[39] G. Rodriguez, Hartley transform: basic theory and applications in oceanographic time series analysis, WIT Trans. on Ecology and the Environment 58 (2002), pp. 319-328, https://www.witpress.com/Secure/elibrary/papers/CENV02/C ENV02030FU.pdf Access date; 18.04.2018].

[40] R. Scott, Doing Hartley smartly, Embedded Systems (2000), pp. 128-144, https://m.eet.com/media/1175816/f-scott.pdf [Access date: 18.04.2018].

[41] G. Schirripa Spagnolo, L. Cozzella, F. Leccese. Viability of an optoelectronic system for real-time roughness measurement, Measurement 58 (2014), pp. 537-543,

DOI: https://doi.org/10.1016/i.measurement.2014.09.018. 